\title{
Irreversible adsorption of latex particles on fibrinogen covered mica
}

\author{
Z. Adamczyk • M. Nattich • M. Wasilewska
}

Received: 15 March 2010 / Accepted: 29 April 2010 / Published online: 15 July 2010

(C) The Author(s) 2010. This article is published with open access at Springerlink.com

\begin{abstract}
Physicochemical properties of bovine plasma fibrinogen $(\mathrm{Fb})$ in electrolyte solutions were characterized. These comprised the diffusion coefficient (hydrodynamic radius), determined by the DLS method, electrophoretic mobility and the isoelectric point. The hydrodynamic radius of $\mathrm{Fb}$ was $12 \mathrm{~nm}$ for $\mathrm{pH}<5$. The number of uncompensated (electrokinetic) charges on the protein $N_{c}$ was calculated from the electrophoretic mobility data. It was found that for $\mathrm{pH}<5.8$ the electrokinetic charge was positive, independently of the ionic strength and negative for $\mathrm{pH}>5.8$. For $\mathrm{pH}=3.5$ the value of $N_{c}$, was 26 for $10^{-3} \mathrm{M}$. Similar electrokinetic measurements were performed for the mica substrate using the streaming potential cell. It was shown that for $\mathrm{pH}=3.5$ and $10^{-3} \mathrm{M}$, the zeta potential of mica remained negative $(-50 \mathrm{mV})$. This promoted an irreversible, electrostatically driven adsorption of $\mathrm{Fb}$, which was confirmed in experiments carried out under diffusion-controlled transport. The surface concentration of Fb on mica was determined directly by AFM counting. By adjusting the time of adsorption, $\mathrm{Fb}$ monolayers of desired coverage were produced. Independently, the presence of $\mathrm{Fb}$ on mica was determined quantitatively by the colloid enhancement method, in which negatively charged latex particles were used, having the diameter of $800 \mathrm{~nm}$. It was found that for $\mathrm{Fb}$ coverage below 0.05 the method was more sensitive than other indirect methods. The experimental data obtained in latex deposition experiments were adequately interpreted in terms of the random site model used previously for polyelectrolytes. It was shown that adsorption sites consisted of a cluster of
\end{abstract}

Z. Adamczyk $(\bowtie) \cdot$ M. Nattich $\cdot$ M. Wasilewska Institute of Catalysis and Surface Chemistry, Polish Academy of Sciences, ul. Niezapominajek 8, 30-239 Cracow, Poland e-mail: ncadamcz@cyf-kr.edu.pl two Fb molecules. It was concluded that the colloid enhancement method can be successfully used for detecting the presence of proteins at solid substrates and to determine the uniformity of monolayers in the nanoscale.

Keywords Fibrinogen monolayers on mica · Colloid enhancement of protein layers - Adsorption of latex on fibrinogen · Irreversible adsorption of colloid particles

\section{Introduction}

Protein adsorption exerts negative effects in processes of artificial organ failure, plaque formation, fouling of contact lenses and heat exchangers, ultrafiltration and membrane filtration units. On the other hand, a controlled protein deposition (irreversible adsorption) on various surfaces is a prerequisite of their efficient separation and purification by chromatography, filtration, for biosensing, bioreactors, immunological assays, etc.

Fibrinogen $(\mathrm{Fb})$ is one of the essential protein playing a key role in the regulation of thrombosis, heamostasis and blood clotting. It is the third most prevalent protein in blood plasma with the concentration $2.6-3 \mathrm{mg} / \mathrm{mL}$ (Agnihotri and Siedlecki 2004) being the most relevant protein that is adsorbed on biomaterial surfaces.

Because of its major significance, numerous experimental works have been devoted to quantify protein, in particular $\mathrm{Fb}$ adsorption on various substrates using the solution depletion methods (Ramsden 1993), the gravimetric methods, especially the quartz microbalance (QCM) (Reisch et al. 2009; Choi et al. 2002), optical methods such as ellipsometry and reflectometry (Melmsten 1994; Buijs et al. 1996, 1997), fluorescence methods such as the total internal fluorescence (TIRF) (Yoon et al. 1996; Wertz and Santore 2001) and isotope labeling (Zembala and Dejardin 1994; Zembala et al. 
1998). For the low coverage range the electron microscopy and the AFM methods can be quite efficient in a direct determination of protein coverage (Hall and Slayter 1959; Veklich et al. 1993; Ortega-Vinuesa et al. 1998; Sit 1999; Marchin and Berrie 2003; Toscano and Santore 2006).

However, despite a significant progress in the field of protein adsorption, there are still discrepancies, even conflicting reports in the literature, concerning the kinetics of this process, maximum coverage, and reversibility aspects. This is mainly caused by a limited availability of direct experimental techniques working under dynamic conditions.

One of few such in situ methods for determining protein adsorption kinetics and properties of their monolayers is based on the streaming potential measurements. Such electrokinetic experiments have been carried out using parallel plate channel cells devised originally by van Wagenen and Andrade (1980) and then used to widely to determine electrokinetic characteristics of bare surfaces (mica, silica slides) (Scales et al. 1990, 1992), colloid particles (Adamczyk et al. 1999; Zembala and Adamczyk 2000; Hayes et al. 1999; Zembala et al. 2003) or protein covered surfaces (Norde and Rouwendal 1990; Elgersma et al. 1992; Vasina and Dejardin 2004; Etheve and Dejardin 2002). However, a disadvantage of the streaming potential measurements is a tedious assembling prior to experiment, sealing problems and the necessity of determining the correction due to surface conductance of the cell.

In this work, we propose another method of detecting protein layers on solid substrates under the wet, in situ conditions. The technique, refereed to as the colloid enhancement (CE), consist in unspecific deposition of larger colloid particles onto protein monolayers under well-controlled transport conditions.

The main goal of our studies is to determine a unique relationship between the amount of adsorbed protein (invisible agent) and the amount of adsorbed colloid, which can be assessed quantitatively via microscope counting. Additionally, important clues on the structure of the protein monolayer, especially density distribution can be gained in a simple way. By inverting this functional dependence, representing a calibration curve, one can determine in a precise way, the unknown amount of adsorbed protein, which can be used to determine their bulk concentration of proteins.

\section{Experimental}

\subsection{Materials}

$\mathrm{Fb}$ from bovine plasma, fraction I, type IV (65\% protein, containing approximately $20 \%$ sodium chloride and $15 \%$ sodium citrate) was purchased from Sigma (F4753) and used without further purification. Protein was in 93\% clottable. The purity of the Fg solution was checked by the dynamic surface tension measurements carried out using the pendant drop shape method. There was practically no change in the surface tension of the $10 \mathrm{ppm}$ solution of Fg within the time of two hours, which is much larger than the typical time of DLS and deposition measurements, lasting 15-30 minutes.

Ruby muscovite mica obtained from Continental Trade was used as substrate, and was freshly cleaved immediately prior to use without any pretreatment.

Water was purified using a Millipore Elix 5 apparatus. Chemicals reagents (buffers, electrolytes) were of analytical grade, and used without further purification. Protein solutions of $\mathrm{Fb}$ concentration in the range of $0.5-50 \mathrm{ppm}$ were prepared as follows: the protein powder was dissolved in $\mathrm{NaCl}$ aqueous solution having the ionic strength of $1 \times 10^{-3}$ or $1 \times 10^{-2} \mathrm{M}$. The $\mathrm{pH}$ of these $\mathrm{Fb}$ solutions was regulated by addition of $\mathrm{HCl}$ or $\mathrm{NaOH}$. Then, the suspension was gently mixed using a magnetic stirrer, and filtered through the Millex ${ }^{\circledR}$-GS $0.45 \mu \mathrm{m}$ filter to eliminate aggregates and impurities. The temperature of experiments was kept constant at $293 \pm 0.1 \mathrm{~K}$.

Positively charged amidine polystyrene latex particles (Invitrogen) having the nominal size of $800 \mathrm{~nm}$ were used as the reference colloid suspension.

On the other hand, deposition studies on Fb monolayers were performed using negatively charged sulfonate polystyrene latex particles (synthesized in our laboratory) having the same nominal size of $800 \mathrm{~nm}$.

\subsection{The experimental methods}

The diffusion coefficient of $\mathrm{Fb}$ under various conditions was determined by dynamic light scattering (DLS), using the Zetasizer Nano ZS Malvern instrument (measurement range of $0.6 \mathrm{~nm}$ to $6 \mu \mathrm{m}$ ). The Nano ZS instrument incorporates noninvasive backscatter $\left(\mathrm{NIBS}^{\mathrm{TM}}\right)$ optics. This technique measures the time-dependent fluctuations in the intensity of scattered light which occur because particles undergo Brownian motion. Analysis of these intensity fluctuations enables the determination of the diffusion coefficients of particles which are converted into a size distribution.

The microelectrophoretic mobility of $\mathrm{Fb}$ was measured using the same Zetasizer Nano ZS Malvern instrument using the Laser Doppler Velocimetry (LDV) technique (measurement range of $3 \mathrm{~nm}$ to $10 \mu \mathrm{m}$ ). In this technique, a voltage was applied across a pair electrode placed at both ends of a cell containing the particle dispersion. Charged particles are attracted to the oppositely charged electrode and their velocity was measured and expressed per unit field strength as the electrophoretic mobility $\mu_{e}$. Then, the zeta potential was calculated using Henry's equation

$\zeta=\frac{3 \eta}{2 \varepsilon F(\kappa a)} \mu_{e}$ 
where $\zeta$ is the zeta potential of proteins, $\mu_{e}$ is the electrophoretic mobility, $\varepsilon$ is the dielectric constant of water, $F(\kappa a)$ is the function of the dimensionless parameter $\kappa a=$ $a / L e, L e=\left(\varepsilon k T / 2 e^{2} I\right)^{1 / 2}$ is the double-layer thickness, $e$ is the elementary charge, $k$ the Boltzman constant, $T$ the absolute temperature, $I=\frac{1}{2}\left(\sum_{i} c_{i} z_{i}^{2}\right)$ is the ionic strength, $c_{i}$ is the ion concentrations, $z_{i}$ is the ion valency and $a$ is the characteristic dimension of a particle or a protein (e.g. its hydrodynamic radius).

AFM measurements of Fb layers were carried out using the NT-MDT Solver PRO device with scanning head SMENA SFC050L. The measurements were performed in semicontact mode using silicon probe (polysilicon cantilevers with resonance frequencies $240 \mathrm{kHz} \pm 10 \%$ or $140 \mathrm{kHz} \pm 10 \%$, typical curvature radius of tip was $10 \mathrm{~nm}$, cone angle was less then $20^{\circ}$ ). The protein solution with $\mathrm{Fb}$ concentration of $0.35 \mathrm{ppm}$ was allowed to adsorb on surface for 5-120 min and then substrate was removed, rinsed for 30 seconds using double distilled water and dried under gentle nitrogen stream.

The real latex particle size as a function of ionic strength and $\mathrm{pH}$ was determined by laser diffractometer (Particle Size Analyzer LS 13320 Beckman Coulter) with an accuracy of few percent and independently by the DLS method using the same Malvern Zetasizer Nano.

Additionally, the size of deposited latex particles was determined by the above AFM device.

The zeta potential of the latex was determined as a function of the ionic strength and $\mathrm{pH}$ using the ZetaPals Brookhaven and the Zetasizer Nano ZS of Malvern.

Streaming potential measurements were carried out using a home-made apparatus described in details previously (Adamczyk et al. 1999, 2006, 2007; Zembala and Adamczyk 2000). The main part of the cell was the parallel plate channel of dimensions $2 b_{c} \times 2 c_{c} \times L$ equal to $0.027 \times$ $0.29 \times 6.2 \mathrm{~cm}$ formed by mica sheets separated by a perfluoroethylene spacer. The electrolyte flow through the channel was forced by a hydrostatic pressure difference $\Delta P$ exerted between in- and outlet compartments. Streaming potential was measured using a pair of $(\mathrm{Ag} / \mathrm{AgCl})$ electrodes connected with an electrometer of high resistance. The other electrode pair (platinium) was used for measuring the electric conductance of the cell. In this way the cell electric resistance $R_{c}$ was determined to incorporate the surface conductivity effect affecting streaming potential in solutions of lower ionic strength. Correction for surface conductivity was introduced according to the procedure described elsewhere (Adamczyk et al. 1999; Zembala and Adamczyk 2000). It is worthwhile mentioning, that this correction was negligible for the ionic strength of the electrolyte above $10^{-3} \mathrm{M}$.

Particle deposition experiments were performed in the diffusion cell of the dimensions $2 \times 2 \times 5 \mathrm{~cm}$, filled up with the latex suspension, having typically the number concentration of $1-2 \times 10^{10} \mathrm{~cm}^{-3}$ and appropriate ionic strength and
$\mathrm{pH}$ value. Wet mica sheets, pre-covered by $\mathrm{Fb}$ monolayers of desired density, were then immersed into the latex suspension. Latex article deposition proceeded over a desired time (typically 24 hours). The true coverage of particles was determined by a direct optical microscope counting under wet conditions, as described in our previous works (Adamczyk et al. 1999, 2006, 2007; Zembala and Adamczyk 2000; Zembala et al. 2003). Particles were counted over 10-20 equal sized areas chosen randomly over the mica sheet. The net number of deposited particles considered was ca. 1500 3000 , which ensures a relative precision of these measurements below $2 \%$, as determined by a variance analysis. It is worthwhile mentioning that the coverage was determined in absolute terms, i.e., $\theta=S_{g}\langle N\rangle$, where $\langle N\rangle$ is the average surface concentration of particles (number of particles per unit area) and $S_{g}=\pi a^{2}$ is the cross-section of the particle ( $a$ is the particle radius). As a consequence, the coverage represents the fraction of the entire surface area occupied by the particles.

However, because of multiple scattering, the optical microscope method, became less accurate for coverage exceeding 0.3. In this case the AFM method (NT MDT Integra system) working in the semi contact mode was used under dry conditions to determine the surface concentration of particles and the coverage.

\section{Results and discussion}

In the first stage of our work, physicochemical characteristics of $\mathrm{Fb}$, latex particles and the mica substrate have been determined, comprising measurements of the diffusion coefficient, microelectrophoretic mobility and the electrokinetic (zeta) potential.

Diffusion coefficient is one of the most important parameters characterizing the dynamics of the protein molecules in solution and their adsorption kinetics on solid substrates. It has been determined directly, as mentioned above, by the DLS method.

It was found that the diffusion coefficient of Fg was practically independent of its bulk concentration, varied within the range 100 to $1000 \mathrm{ppm}$ and assumed an average value of $2.1 \times 10^{-7} \mathrm{~cm}^{2} \mathrm{~s}^{-1}$ for ionic strength range $10^{-2}-0.15 \mathrm{M}$ $(\mathrm{pH}=3.5-5)$. This suggests that for this $\mathrm{pH}$ and ionic strength range, there were no specific effects stemming from protein aggregation and interactions in the bulk.

From the diffusion coefficient measurements one can determine the Stokes hydrodynamic radius of the protein using the known dependence (Adamczyk et al. 2010)

$R_{H}=\frac{k T}{6 \pi \eta D}$

where: $R_{H}$ is the hydrodynamic radius, $D$-the diffusion coefficient and $\eta$-the dynamic viscosity of the solution. 
The advantage of using $R_{H}$ is that it is independent of the temperature and viscosity of the suspending medium. This parameter can also be used for a quantitative assessment of protein aggregation phenomena, which induce an increase in $R_{H}$.

It was calculated from (2), using the above $\mathrm{Fb}$ diffusion coefficient value, that measured experimentally, that $R_{H}=12 \mathrm{~nm}$ (at the temperature of $293 \mathrm{~K}$ ). It is interesting to observe that this is much higher than the radius of the equivalent sphere (having the same volume as the hydrated $\mathrm{Fb}$ molecule equal to $440 \mathrm{~nm}^{3}$, see Table 1) which reflects the very elongated shape of $\mathrm{Fb}$ determined from crystallographic data (see Table 1).

In further analysis we approximate the complex shape of Fb by the bead model, used previously to determine its adsorption kinetics on solid substrates (Adamczyk et al. 2010). According to this model the molecule is replaced by a string of 23 co-linear touching spheres of various diameters (see Table 1). The two external spheres have the diameter of $6.7 \mathrm{~nm}$ and the central sphere has the diameter of $5.3 \mathrm{~nm}$. All remaining 20 spheres have the diameter of $1.5 \mathrm{~nm}$. The overall length of the molecule is $48.7 \mathrm{~nm}$, its cross-section area $S g$ (in the plane parallel to the symmetry axis of the $\mathrm{Fb}$ molecule) equals $128 \mathrm{~nm}^{2}$ and its volume is $440 \mathrm{~nm}^{3}$.

Another parameter determined in order to characterize $\mathrm{Fb}$ dynamics was the electrophoretic mobility $\mu_{e}$. It is defined as the average translation velocity of the protein $V$ under a given electric field $E$. This quantity is measured directly by microelectrophoresis, as described above.

Knowing the electrophoretic mobility of a particle, one can calculate the averaged number of uncompensated (free) charges per molecule $N_{c}$ from the Lorenz-Stokes relationship (Wasilewska et al. 2009):

$N_{c}=\frac{6 \pi \eta \cdot 10^{8}}{1.602} R_{H} \mu_{e}$

where $N_{c}$ is expressed in elementary charges $e=1.602 \times$ $10^{-19}[\mathrm{C}], \mu_{e}$ is expressed in $\left[\mu \mathrm{m} \mathrm{cm} \mathrm{s}^{-1} \mathrm{~V}^{-1}\right]$, and $\mathrm{V}$ is the volt unit.

It is interesting to observe that (3) is valid for arbitrary shape of particles.

The dependence of $N_{c}$ on $\mathrm{pH}$ for $\mathrm{Fb}$ is plotted in Fig. 1. As can be seen, for $\mathrm{pH}>5.8, N_{c}$ attained negative values decreasing monotonically with $\mathrm{pH}$. Thus $N_{c}=-29$ for $\mathrm{pH}=9$.

On the other hand, for $\mathrm{pH}<5.8, N_{c}$ became positive, attaining for $3<\mathrm{pH}<4$ and $I=10^{-3}-10^{-2} \mathrm{M}$, values between 32 and 23 (the electrophoretic mobility varied between 2.2 and $1.5 \mu \mathrm{m} \mathrm{cm} \mathrm{s}^{-1} \mathrm{~V}^{-1}$, respectively). As can be seen, the number of positive charges per one Fg molecule is significant for this $\mathrm{pH}$ and ionic strength range. This indicates unequivocally that for $\mathrm{pH}<5.8 \mathrm{Fb}$ is likely to adsorb on negatively charged surfaces, e.g., mica, according to the side-on mechanism.

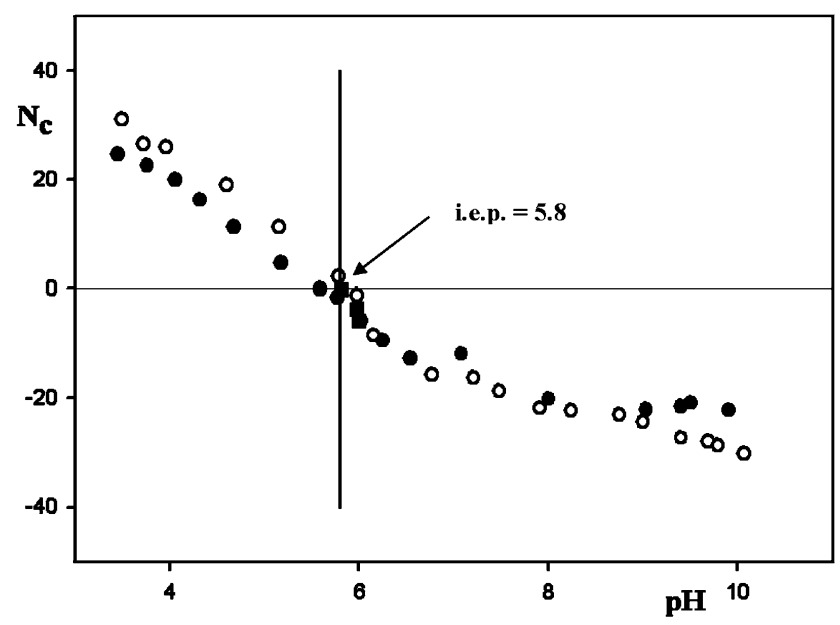

Fig. 1 The dependence of the number of uncompensated charges per one $\mathrm{Fb}$ molecule $N_{c}$ (expressed in elementary charges $e$ ) on $\mathrm{pH}$ determined from microelectrophoretic measurements. $1 . I=1 \times 10^{-3} \mathrm{M}$ $\mathrm{NaCl}$ (circles-O). 2. I=1 $\times 10^{-2} \mathrm{M} \mathrm{NaCl}$ (full circles-

In an analogous way the hydrodynamic radius and microelectrophoretic mobility of latexes used in our studies have been characterized. The average hydrodynamic radius of the A800 latex for the $\mathrm{pH}$ range 3-6 was $400 \mathrm{~nm}$ (ionic strength $10^{-3} \mathrm{M}$ ) and for the $\mathrm{L} 800$ latex $405 \mathrm{~nm}$, for the same range of $\mathrm{pH}$ and ionic strength. Because latex particles were spherical in shape (see Fig. 2, where the AFM micrograph of latex particles on mica is shown), the latex particle diameter equals $2 R_{H}=800 \mathrm{~nm}$ for the A800 and $810 \mathrm{~nm}$ for the L800 sample.

The electrophoretic mobility of latex was determined analogously as for $\mathrm{Fb}$. For sake of convenience it was converted to the zeta potential $\zeta_{p}$ data, which are commonly used to characterize colloid suspensions. The results showing the dependence of both latexes zeta potential $\zeta_{p}$ on $\mathrm{pH}$ at fixed ionic strength of $I=10^{-3} \mathrm{M}, \mathrm{NaCl}$ are plotted in Fig. 3. As can be noticed, the A800 (positive latex) particles zeta potential was independent of $\mathrm{pH}$ (changed within the range 3-9) assuming an average value of $75 \mathrm{mV}$ (for $I=10^{-3} \mathrm{M}$ ) and $95 \mathrm{mV}$ for $I=10^{-2} \mathrm{M}$.

The zeta potential of the L800 latex was also fairly independent of $\mathrm{pH}$ (changed within the range 3-9) assuming an average value of $-80 \mathrm{mV}$ (for $I=10^{-3} \mathrm{M}$ ) and $-105 \mathrm{mV}$ for $I=10^{-2} \mathrm{M}$.

The zeta potential of the mica substrate was determined via the streaming potential measurements according to the procedure described above. The results obtained in the case of $\mathrm{NaCl}$ electrolyte are shown in Fig. 4. As can be seen, at a constant ionic strength of $10^{-3} \mathrm{M}$ the zeta potential of mica decreased systematically with the increase in $\mathrm{pH}$, varying between $-50 \mathrm{mV}$ for $\mathrm{pH}=3.5,-85 \mathrm{mV}$ at $\mathrm{pH}=5.5$ and $-118 \mathrm{mV}$ for $\mathrm{pH} 10$. As can be seen this behavior can be reasonably well reflected by the theoretical model (depicted by the solid line in Fig. 4), formulated by assuming a com- 
Table 1 Physicochemical properties of fibrinogen

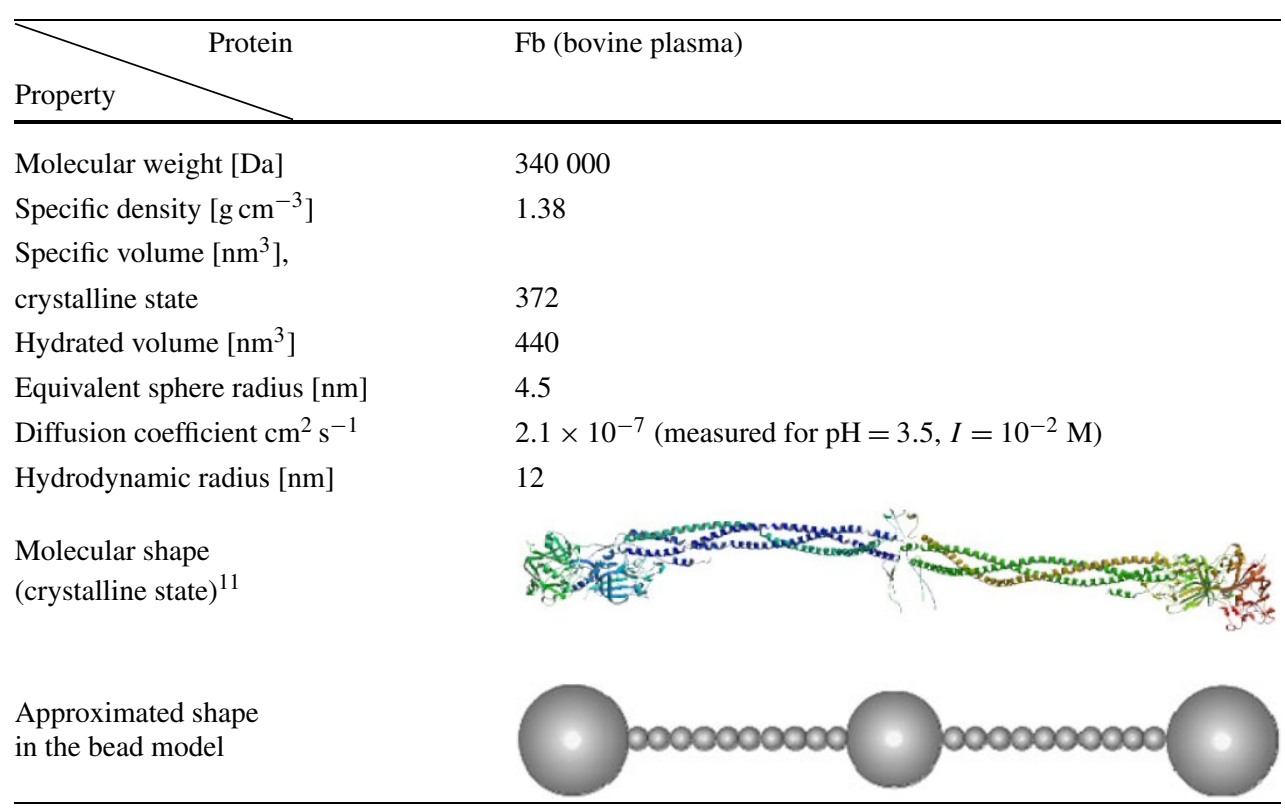

Fig. 2 The topology of the A800 and L800 latex samples on mica and mica modified by $\mathrm{Fb}$ determined by AFM
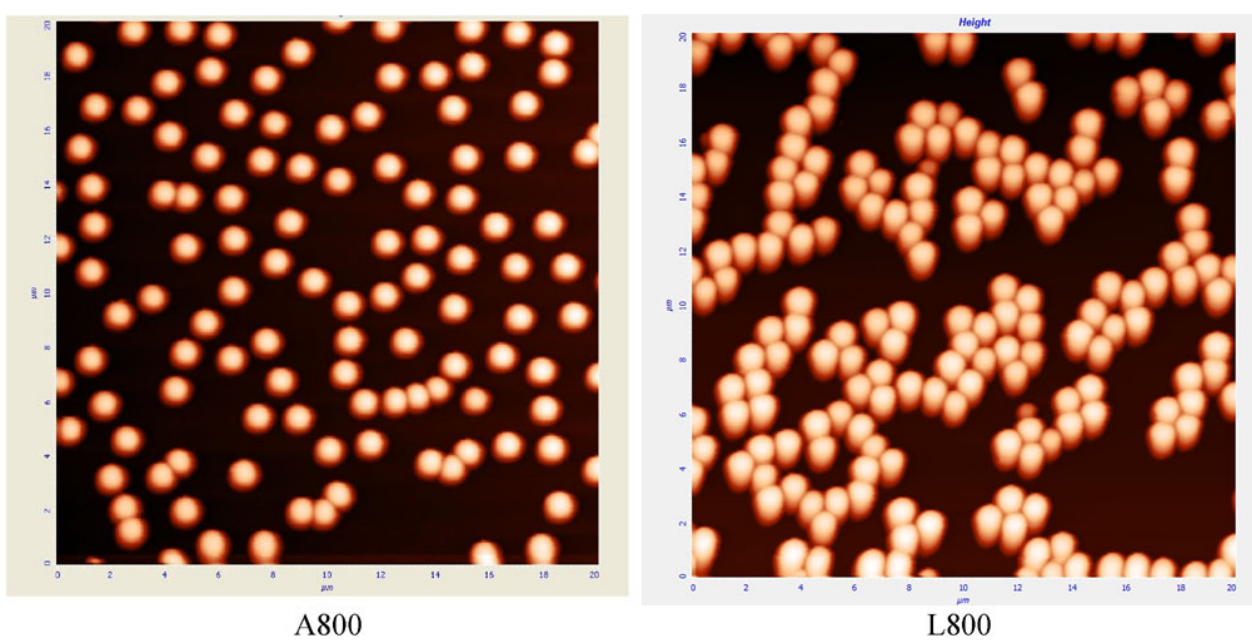

petitive adsorption of the $\mathrm{H}^{+}$and $\mathrm{Na}^{+}$cations on the surface binding sites at mica (Adamczyk et al. 2010).

These physicochemical measurements suggest that $\mathrm{Fb}$ is expected to adsorb strongly on mica for the $\mathrm{pH}<5.8$, due to attractive electrostatic interactions. This can lead to formation of $\mathrm{Fb}$ monolayers, whose density (coverage) can be well controlled by the adsorption time. In order to verify this hypothesis, systematic $\mathrm{Fb}$ adsorption experiments have been conducted, under the diffusion-controlled transport conditions. The dependence of the surface concentration of $\mathrm{Fb} N$ (expressed on $\mu^{-2}$ ) on the adsorption time $t$ was determined in these experiments. Results of these measurements performed for $\mathrm{Fb}$ concentration of $0.35 \mathrm{ppm}$, $\mathrm{pH}=3.5, I=10^{-3} \mathrm{M}$ and $T=293 \mathrm{~K}$ are shown Fig. 5 . The surface concentration of $\mathrm{Fb}$ was determined by direct counting of the number of molecules per unit area of AFM images, typically having the size of $2 \times 2 \mu \mathrm{m}$. Be- cause of the AFM resolution limitations, the highest $N$ value accessible in these experiments was $300 \mu \mathrm{m}^{-2}$. It is to mention that results shown in Fig. 5 represent, according to our knowledge, first quantitative data reported in the literature on $\mathrm{Fb}$ side-on adsorption under diffusion controlled transport conditions, derived using a direct observation method.

As can be seen, the experimental data are well reflected by theoretical results, described by the following dependence (Adamczyk et al. 2010; Adamczyk 2006)

$N=2\left(\frac{D t}{\pi}\right)^{1 / 2} n_{b}$

where $n_{b}=c_{p p m} A v \times 10^{-6} / M_{w}$ is the number concentration of $\mathrm{Fb}$ molecules in the bulk ( $c_{p p m}$ is the $\mathrm{Fb}$ concentration in ppm, $A v$ is the Avogadro constant). 
Fig. 3 The dependence of the zeta potential of polystyrene latex particles $\zeta_{p}$ on $\mathrm{pH}$, $T=293 \mathrm{~K}$. The points denote experimental values determined for 1 . (๑) $I=10^{-2} \mathrm{M}, \mathrm{NaCl}$ (positive latex A800), 2. (O) $I=10^{-3} \mathrm{M}, \mathrm{NaCl}$ (positive latex A800), 3. ( $\Delta) I=10^{-3} \mathrm{M}$, $\mathrm{NaCl}$ (negative latex L800), 4. $(\triangle) I=10^{-2} \mathrm{M}, \mathrm{NaCl}$ (negative latex L800)

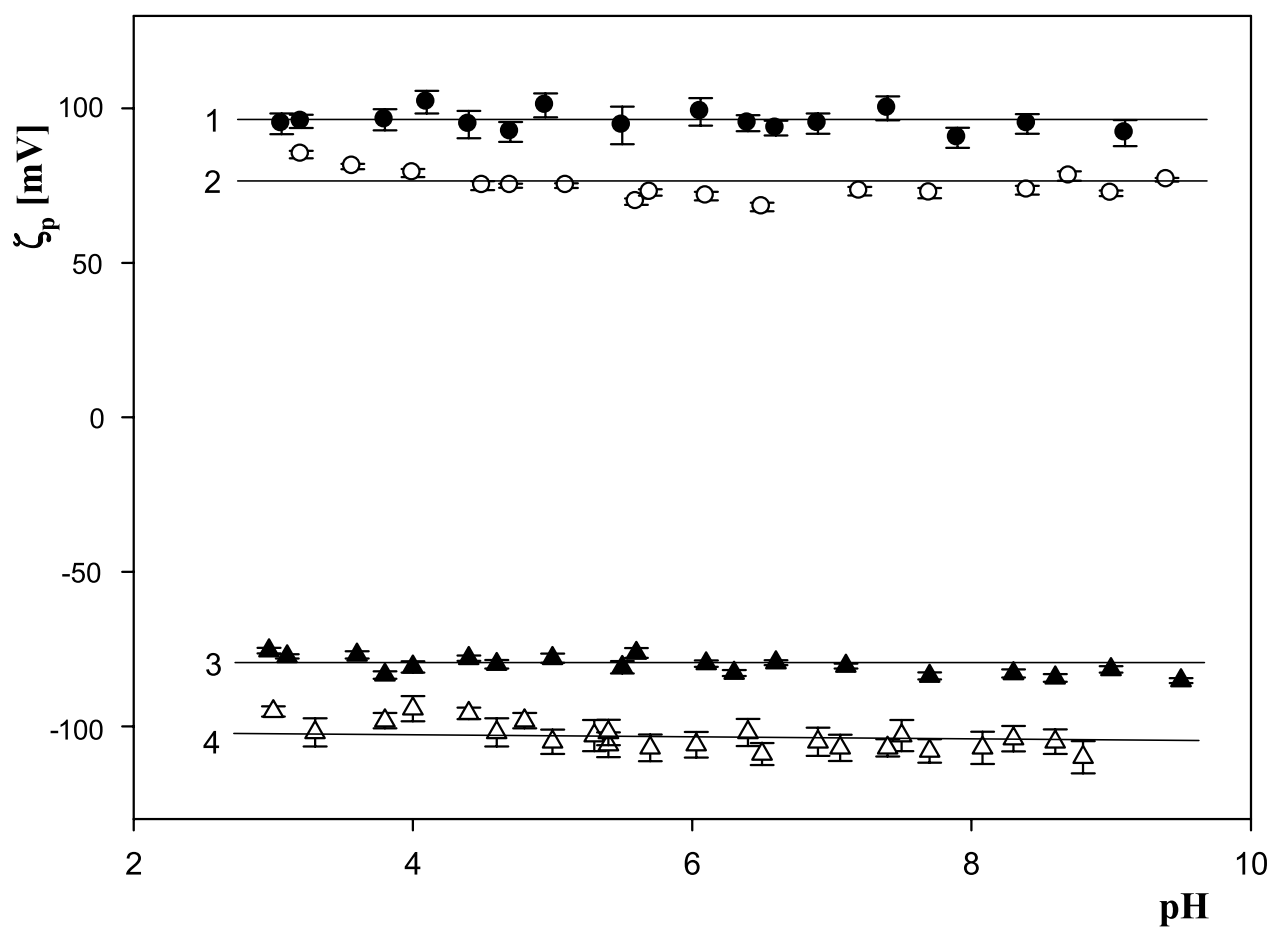

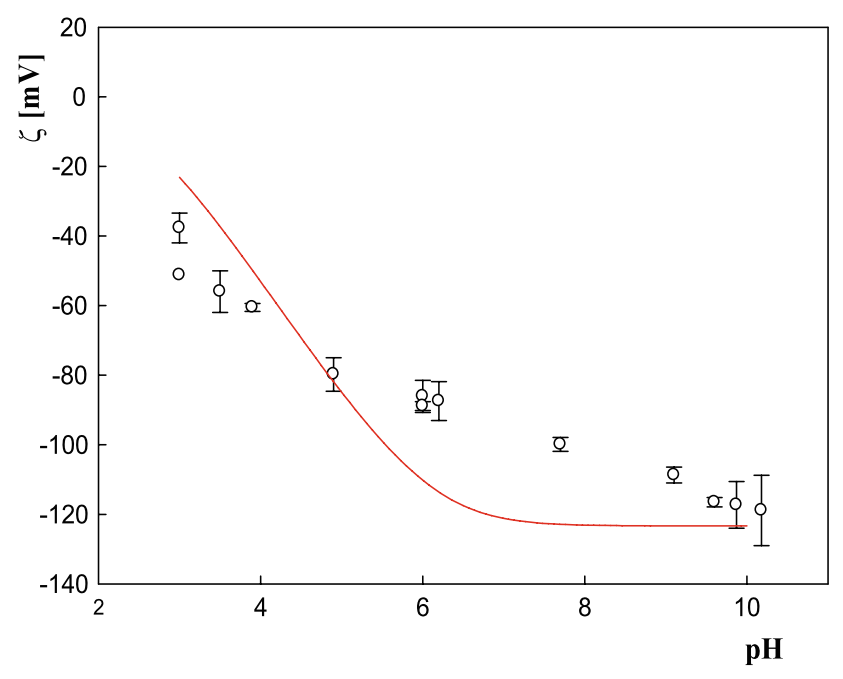

Fig. 4 The dependence of the zeta potential of mica $\zeta[\mathrm{mV}]$ on $\mathrm{pH}$ determined by the streaming potential measurements in the parallel-plate cell. The points denote the experimental results obtained for $I=1 \mathrm{mM}$ and the solid line shows the theoretical results derived from the site binding model

It was demonstrated in Adamczyk et al. (2010) that the linear dependence described by (4) remains valid for $N$ reaching the jamming limit, which was determined to be $2.26 \times 10^{3} \mu \mathrm{m}^{-2}$. It is interesting to observe that the maximum coverage, which corresponds to this $N$ value is $\Theta=N S_{g}=0.29$.

Therefore, one can extrapolate the kinetic dependence shown in Fig. 5 to longer times, obtaining the coverage of
$\mathrm{Fb}$, which can be used for interpretation of latex particle deposition.

In the first stage of these latex deposition studies, the reference data for bare homogenous surfaces were obtained. The goal of these measurements was to determine the full kinetic curve of latex on bare mica (uncovered by $\mathrm{Fb}$ ), in particular the maximum coverage, used later on as the reference variable for scaling the curves obtained for Fb layers. The kinetic curve obtained for the A800 latex under diffusion-controlled transport for $\mathrm{pH}=3.5, I=10^{-3} \mathrm{M}$, $n_{b}=1.77 \times 10^{10}$ is shown in Fig. 6 as the dependence of the latex coverage $\Theta_{L}$ on the square of the deposition time $t^{\frac{1}{2}}$. As can be seen, this dependence remains linear for $\Theta_{L}<0.3$, which is in accordance with (4), where the latex coverage was calculated as $\Theta_{L}=\pi a^{2} N_{L}$ (where $N_{L}$ is the latex surface concentration). However, for higher latex coverage, significant deviations from linearity occurred, and the kinetic curve approached gradually the limiting value $\Theta_{m x}=0.46$. These deviations from linearity are caused by the surface blocking effects, which were analyzed in detail in Adamczyk et al. (1999, 2010), Adamczyk (2006), Schaaf and Talbot (1989). It was shown that the blocking function $B(\Theta)$ in the case of spherical particles has the form

$$
\begin{aligned}
B(\Theta)= & {\left[1+0.812 \frac{\Theta}{\Theta_{m x}}+0.4258\left(\frac{\Theta}{\Theta_{m x}}\right)^{2}\right.} \\
& \left.+0.0716\left(\frac{\Theta}{\Theta_{m x}}\right)^{3}\right]\left(1-\frac{\Theta}{\Theta_{m x}}\right)^{3}
\end{aligned}
$$




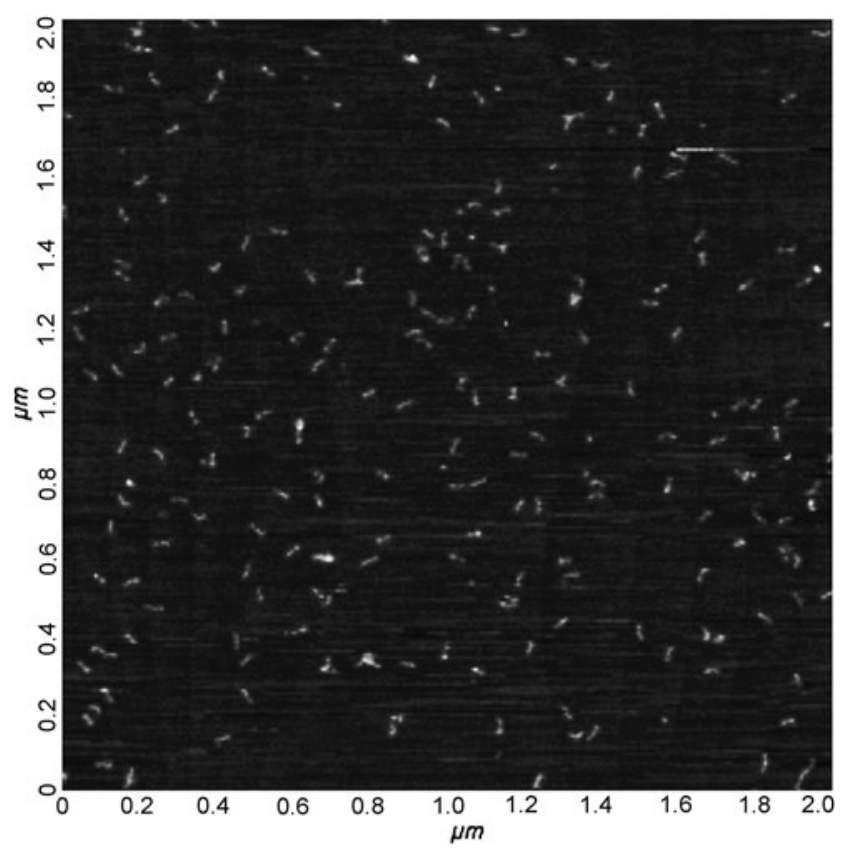

(a)

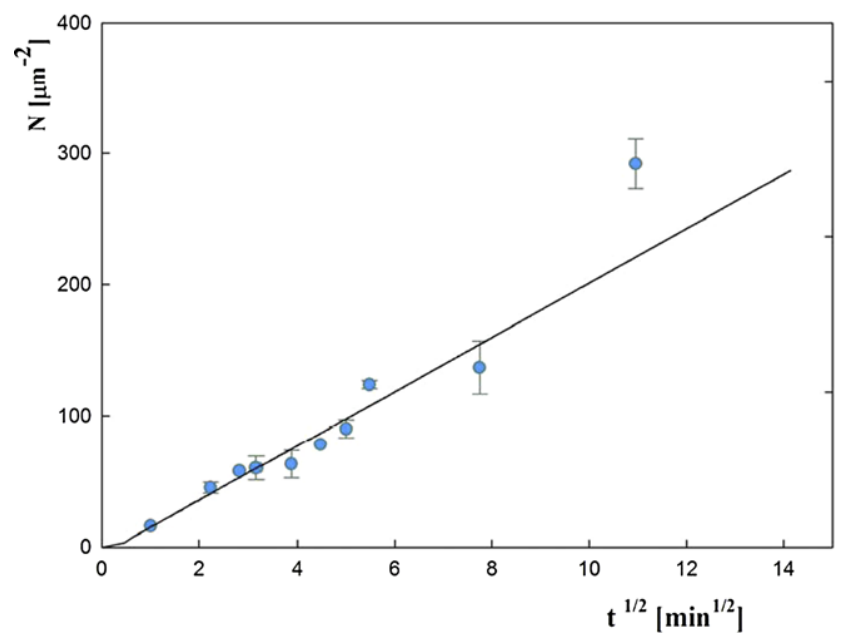

b

Fig. 5 (a) The topology of the Fb monolayer on mica surface determined by AFM semi-contact mode, for $\mathrm{pH}=3.5, T=293 \mathrm{~K}$, $I=10^{-3} \mathrm{M}$ and the surface concentration of $\mathrm{Fb} N\left[\mu \mathrm{m}^{-2}\right]$ equal to 47 . (b) The dependence of the surface concentration of $\mathrm{Fb} N\left[\mu \mathrm{m}^{-2}\right]$ on the square root of the adsorption time $\left.t^{1 / 2} \mathrm{~min}^{1 / 2}\right]$. The points denote the experimental results obtained for $\mathrm{pH}=3.5, T=293 \mathrm{~K}, I=10^{-3} \mathrm{M}$ using the AFM topological measurements and the solid line shows the theoretical results calculated from the generalized diffusion model (Adamczyk 2000)

where the maximum coverage $\Theta_{m x}$ for hard (non-interacting) particles, which is referred to as the jamming coverage equals 0.547 (Hinrichsen et al. 1986).

However, as can be seen in Fig. 6, the maximum coverage was smaller because of the appearance of lateral electrostatic repulsion between adsorbing latex particles. Using the effective interaction range concept, it was shown (Adamczyk et

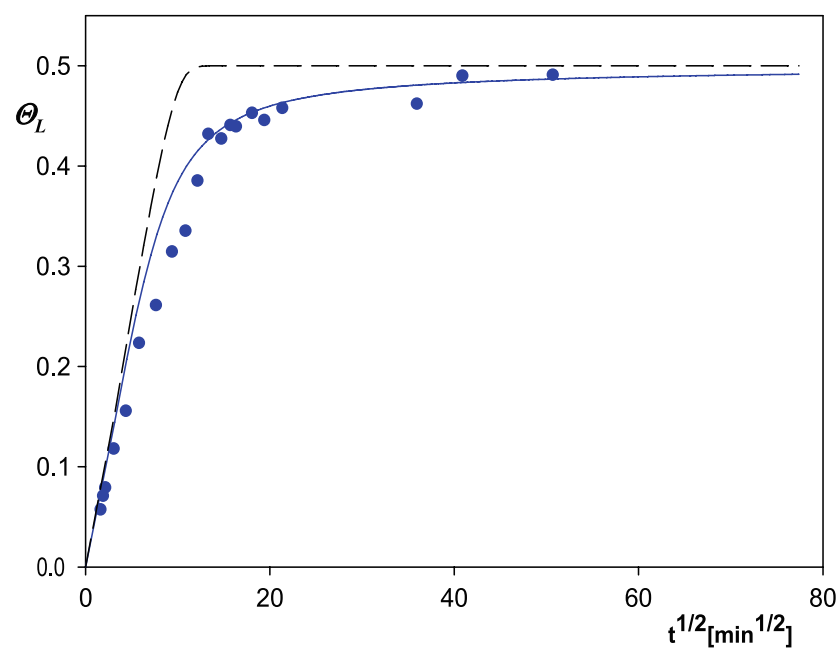

Fig. 6 Deposition kinetics of positively charged polystyrene latex particles (averaged diameter of $800 \mathrm{~nm}$ ) on mica, determined by optical microscopy (circles- - ), for the ionic strength $10^{-3} \mathrm{M}, \mathrm{pH}=3.5$, $T=298 \mathrm{~K}, n_{b}=1.77 \times 10^{10} \mathrm{~cm}^{-3}$. The solid line denotes exact theoretical results calculated by solving the governing diffusion equation with the blocking effect given by the RSA model, and the dashed line represents the theoretical results calculated using the Langmuir model

al. 2010; Adamczyk 2006) that the maximum coverage can be calculated in this case from the dependence

$\Theta_{m x}=\Theta_{\infty} \frac{1}{\left(1+h^{*} / a\right)^{2}}$

where $h^{*}$ is the effective interaction range, which can be approximated by the analytical dependence (Adamczyk et al. 2010)

$h^{*} / a=\frac{1}{2\left(\frac{a}{L_{e}}\right)}\left\{\ln \left(\frac{\phi_{0}}{2 \phi_{c h}}\right)-\ln \left[1+\frac{1}{2\left(\frac{a}{L_{e}}\right)} \ln \left(\frac{\phi_{0}}{2 \phi_{c h}}\right)\right]\right\}$

where $\phi_{0}$ is the repulsive interaction energy between particle pair at close separations (height of the energy barrier), and $\phi_{c h}$ is the characteristic energy close to $1 \mathrm{kT}$ (Adamczyk 2006).

By knowing the blocking function and the maximum coverage one can calculate theoretically particle adsorption kinetics by numerical integration of the governing diffusion equations with the boundary condition at the interface having the form (Adamczyk 2000, 2006)

$j(\Theta, t)=k_{a} n\left(\delta_{a}\right) B(\Theta)$

where $j(\Theta, t)$ is particle flux at the interface, $n\left(\delta_{a}\right)$ is the local particle concentration at the edge of the adsorption layer of the thickness $\delta_{a}$ (which can be identified with the particle diameter $2 a$ ).

As can be seen in Fig. 6, the theoretical results obtained by solving the bulk transport equation using the implicit, 
finite-difference method of Crank-Nicholson (solid line), are in a good agreement with experimental data for the entire range of adsorption time reaching 30 hours. On the other hand, the results obtained by using the Langmuir blocking function in the form $B(\Theta)=1-\Theta / \Theta_{m x}$ proved inadequate (see the dashed line in Fig. 6).

It is interesting to observe, that due to symmetry of the electrostatic interaction equation, the same results are obtained in the case where the latex particles bear a negative zeta potential and the interface a positive zeta potential, provided that the size of latex particles remains the same. Therefore, the results shown in Fig. 6, obtained for homogeneous surfaces, can be used as convenient reference system for analyzing deposition of negative latex particles of the same size on heterogeneous surfaces produced by a controlled $\mathrm{Fb}$ adsorption. In the first stage of these experiments, a $\mathrm{Fb}$ monolayer on mica of a desired coverage (determined by direct AFM measurements) was produced. Then, the wet mica sheets were placed in the diffusion cell and latex deposition was carried out for 24 hours. After rinsing in flow conditions (30 seconds in double distilled water) the latex coverage was determined without drying mica sheet by optical microscopy. This procedure allowed one to avoid distortions of the $\mathrm{Fb}$ and latex monolayers, which is often the case when drying procedure is applied prior to microscope observations. In this way, we can determine in a reproducible way not only the latex coverage but the homogeneity of particle distributions over the mica substrate, analyzed in terms of the pair correlation function.

The results of the latex deposition experiments (L800 sample) on Fb covered mica are shown in Fig.7 in the form of the dependence of $\Theta_{L}$ on the Fb coverage $\Theta_{f}$. The conditions of these experiments were the same as for the calibration experiments involving the A800 latex sample, i.e., $\mathrm{pH}=3.5, I=10^{-3} \mathrm{M}, n_{b}=1.77 \times 10^{10}$. As can be seen, the latex coverage increased abruptly with $\Theta_{f}$, attaining $0.8 \Theta_{m x}$ for $\Theta_{f}$ as low as $0.05(5 \%)$. Thus, the initial slope of the $\Theta_{L}$ vs. $\Theta_{f}$ dependence exceeded 20, which indicated that the sensitivity of detecting Fb layers by latex deposition was rather high.

However, as can be seen in Fig. 7, for $\Theta_{f}=0.05$, the increase in the latex coverage with the $\mathrm{Fb}$ coverage was less steep, which prohibits an exact evaluation of $\mathrm{Fb}$ coverage using latex deposition data.

It is interesting to interpret the experimental data shown in Fig. 7 theoretically since such colloid enhancement experiments have not been done before in the literature. We applied the random site method, used previously to interpret particle deposition on surfaces covered by polyelectrolyte molecules (Adamczyk et al. 2006, 2007). The basic parame-

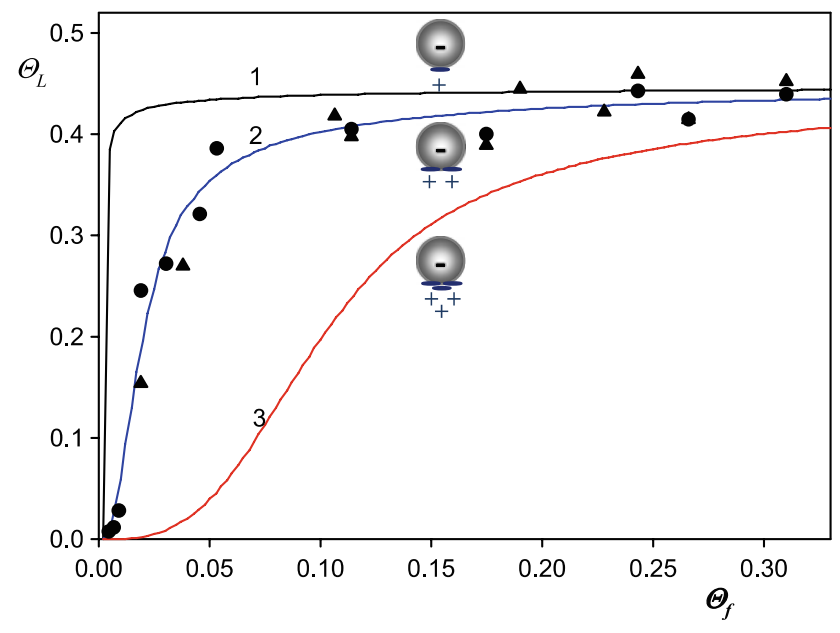

Fig. 7 The dependence of the maximum coverage of negative latex particles $\Theta_{L}$ on the coverage of $\mathrm{Fb}, \Theta_{f}$. The points denote the experimental results obtained by optical microscopy (circles-0) and $\operatorname{AFM}$ (triangles- $\mathbf{\Delta}$ ), for ionic strength $10^{-3} \mathrm{M}, \mathrm{pH}=3.5, T=298 \mathrm{~K}$, $n_{b}=1.77 \times 10^{10} \mathrm{~cm}^{-3}$ deposition time 20 hours. The solid lines $1-3$ denote theoretical prediction derived from (10), (14) for the adsorption site composed of one two and three $\mathrm{Fb}$ molecules, respectively

ter of this approach, characterizing the density of adsorption sites, is defined as

$\alpha=\pi a^{2} N_{s}=\frac{\pi a^{2}}{S_{g_{s}}} \Theta_{s}=\lambda^{2} \Theta_{s}$

where $N_{s}$ is the surface concentration of adsorption sites, $S_{g_{s}}$ is the characteristic cross-section area of one site and $\lambda=\left(\frac{\pi a^{2}}{S_{g s}}\right)^{1 / 2}$.

Assuming that in our case the sites are formed by single $\mathrm{Fb}$ molecules having the cross-section area of $1.28 \times$ $10^{-12} \mathrm{~cm}^{2}$ (see Table 1) and $\pi a^{2}=5.03 \times 10^{-9} \mathrm{~cm}^{2}$ one obtains $\lambda^{2}=3.93 \times 10^{3}$. Moreover, in our case $\Theta_{s}=\Theta_{f}$.

Knowing the $\alpha$ parameter one can calculate the maximum coverage of particles on a heterogeneous surface covered by sites from the analytical dependence (Adamczyk 2006; Jin et al. 1993)

$\Theta_{L}=\Theta_{m x}\left(1-\frac{1+0.314 \alpha^{2}+0.45 \alpha^{3}}{1+1.83 \alpha+0.66 \alpha^{3}+\alpha^{7 / 2}}\right)$

In the limit of low coverage of sites, where $\alpha \ll 1$, (10) simplifies to

$\Theta_{L}=\lambda^{2} \Theta_{S}$

As can be seen, it is predicted in this case that the latex coverage increases linearly with the site coverage.

On the other hand, for larger site coverage range, where $\alpha \gg 1,(10)$ reduces to the limiting form

$\Theta_{L}=\Theta_{m x}\left(1-\frac{0.45}{\alpha^{1 / 2}}\right)=\Theta_{m x}\left(1-\frac{0.45}{\lambda \Theta_{s}^{1 / 2}}\right)$ 
Thus, for large site coverage, the maximum latex coverage is approached proportionally to $\Theta_{s}^{1 / 2}$.

As can be seen in Fig. 7, the theoretical results derived from (10) (curve 1) do not reflect properly the experimental data, since they predict a much steeper increase of $\Theta_{L}$ with the $\mathrm{Fb}$ coverage (the slop should be $\lambda^{2}=3.93 \times 10^{3}$ as estimated above) than experimentally observed. This indicates that adsorption sites consisting of one $\mathrm{Fb}$ molecule were not effective to immobilize the latex particle of much larger dimensions. Analogous phenomenon was observed previously in the case poly(allylamine) hydrochloride and poly(ethylene imine) polyelectrolytes (Adamczyk et al. 2006, 2007), where latex particle deposition occurred at adsorption centers composed of two or more closely spaced polyelectrolyte chains. It was shown, assuming the Poisson statistics that the coverage of adsorption sites composed of $n_{s}$ or more particles is given by the expression (Adamczyk et al. 2006, 2007)

$\Theta_{s}\left(n_{s}\right)=e^{-\bar{S} \Theta_{f}} \sum_{n=n_{s}}^{\infty} \frac{\left(\bar{S} \Theta_{f}\right)^{n}}{n} \cong \frac{\left(\bar{S} \Theta_{f}\right)^{n_{s}}}{n_{s} !}$

where $\bar{S}=S^{*} / S_{g}$ and $S^{*}$ is the effective interaction area, whose size is comparable with the cross-section area of the site, thus $S^{*} \approx 1$.

As can be noticed, the coverage of sites composed of two $\mathrm{Fb}$ molecules is proportional to $\Theta_{f}^{2}$, three molecules $\Theta_{f}^{3}$, etc. This means that the number of such sites is very low for $\mathrm{Fb}$ coverage not exceeding 0.1. Therefore, using (13) and (11) one can predict that the latex coverage increases initially according to the relationship

$\Theta_{L}=\lambda^{2} \frac{\Theta_{f}^{n_{s}}}{n_{s} !}$

Thus, in the case of $n_{s}=2$, a parabolic dependence of $\Theta_{L}$ on the $\mathrm{Fb}$ coverage is predicted initially.

For arbitrary range of $\mathrm{Fb}$ coverage, (10) can be used. As can be seen in Fig. 8, the experimental data are well reflected by theoretical predictions derived from this equation, for the entire range of $\mathrm{Fb}$ coverage. In particular, the parabolic dependence for low $\Theta_{f}$ coverage was confirmed. Also the approach to the maximum coverage can be seen, proportional to $\lambda^{2} \Theta_{s}^{-1 / 2} \cong \lambda^{2} \Theta_{f}^{-1}$ was confirmed.

On the other hand, the theoretical results calculated by assuming that the adsorption site was composed of three $\mathrm{Fb}$ molecules (curve 3 in Fig. 7) proved inadequate, since they underestimated significantly the experimental data.

Additional information on the uniformity and $\mathrm{Fb}$ monolayers can be derived analyzing statistically the distribution of deposited latex particles using the micrographs obtained by optical microscopy. An example of such a micrograph of latex monolayer taken for $I=1 \times 10^{-3} \mathrm{M}, \Theta_{f}=0.12$,

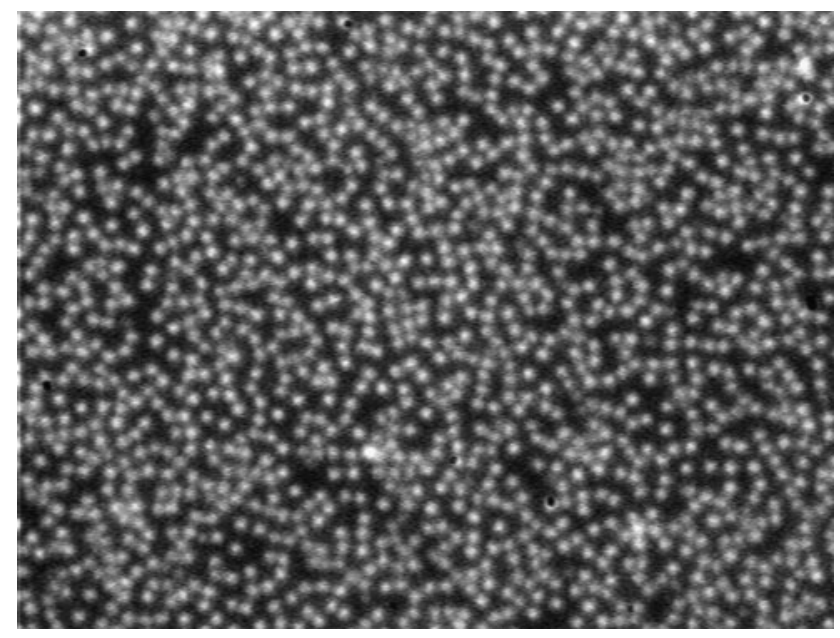

(a)

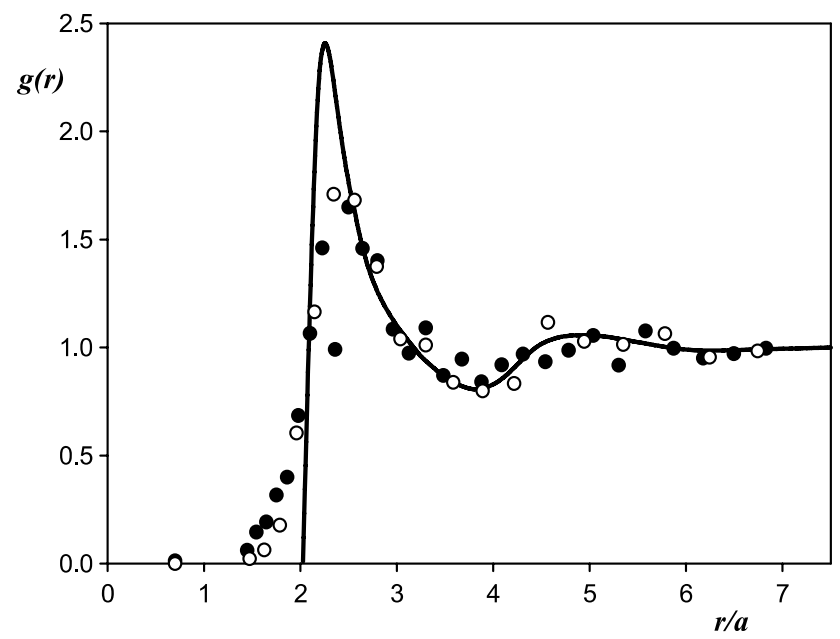

(b)

Fig. 8 (a) Micrographs showing positively charged latex particles (averaged diameter $800 \mathrm{~nm}$ ) deposited on the $\mathrm{Fb}$ layer on mica, $\Theta_{f}=0.12, \Theta_{L}=0.44$. (b) The pair correlation function $g(r)$ for the latex particles. The points denote experimental results derived from optical microscopy for: latex particles adsorbing on bare mica $\Theta_{L}=0.44$, $\bigcirc$ latex particles adsorbing on a $\mathrm{Fb}$ layer bare mica $\Theta_{f}=0.12, \Theta_{L}=0.44$. The solid line denotes the theoretical results derived from the Monte-Carlo RSA simulations

$\Theta_{L}=0.44$ is shown in Fig. 8a. A short-range, liquid-like ordering of particles can be clearly observed in this picture. It is also interesting to mention that the latex monolayer was statistically uniform, which was confirmed by a variance analysis carried out for various areas (of the same size) chosen randomly over the monolayer.

Quantitatively, the structure of particle monolayers can be characterized in terms of the pair correlation function $g(r / a)$ (often referred to as the radial distribution function) (Adamczyk et al. 1990, 2010; Adamczyk 2006; Schaaf and Talbot 1989; Hinrichsen et al. 1986). This function expresses the ensemble averaged probability of finding a particle pair at the center to center distance $r$ (normalized usually 
to particle radius $a$ ). Such pair correlation function was determined by analyzing positions of ca 2000 latex particles within the monolayer shown in Fig. 8a. The pair correlation function determined in this way is shown in Fig. 8, part "b". As can be seen, a characteristic feature of the $g(r / a)$ function shown that it is exhibited a well pronounced maximum at the distance $r / a$ close to $2.1 a$. The position of the peak is a direct indication of the range of the lateral interaction range $2 h^{*}$ among adsorbed and adsorbing particles. Indeed, using (7) one can predict that $2 h^{*}=0.12$, which agrees well with the peak position.

It is also interesting to observe that the pair correlation function obtained for latex particles on the heterogeneous mica substrate was identical, within the experimental error, with the function determined for homogeneous surfaces (full points in Fig. 8b). Additionally, these experimental data were adequately interpreted in terms of theoretical results derived from Monte-Carlo type simulation (Adamczyk et al. 2010; Adamczyk 2006), depicted by the solid line in Fig. 8b. These simulation were performed using the random sequential adsorption model for continuous surfaces. Therefore, this agreement between experimental data obtained for homogeneous and heterogeneous surfaces, and with theoretical results suggests unequivocally that $\mathrm{Fb}$ monolayers on mica were statistically uniform in the microscale.

\section{Concluding remarks}

$\mathrm{Fb}$ was adsorbing irreversibly on mica substrate for $\mathrm{pH}=$ 3.5 and the ionic strength of $10^{-3} \mathrm{M}$ due to electrostatic attraction, which was confirmed by direct AFM measurements. This process was controlled by the diffusion of $\mathrm{Fb}$ molecules from the bulk rather than by surface activation barriers.

The presence of $\mathrm{Fb}$ was quantitatively determined by the colloid enhancement method, in which negatively charged latex particles were used. The precision of these measurements was especially high for the low Fb coverage, $\Theta_{f}<$ 0.05 .

The dependence of the latex coverage on the density of the $\mathrm{Fb}$ monolayer was quantitatively interpreted in terms of the random site model, by assuming that the site consisted of the cluster of two $\mathrm{Fb}$ molecules.

The experiments performed in this work showed that the colloid enhancement method can be effectively used for detecting the presence of proteins at solid substrates and to determine the uniformity of their monolayers in the microscale.

Acknowledgements This work was supported by the COST Action D43 Special Grant.
Open Access This article is distributed under the terms of the Creative Commons Attribution Noncommercial License which permits any noncommercial use, distribution, and reproduction in any medium, provided the original author(s) and source are credited.

\section{References}

Adamczyk, Z.: J. Colloid Interface Sci. 220, 477 (2000)

Adamczyk, Z.: Particles at Interfaces: Interactions, Deposition, Structure. Academic Press/Elsevier, New York (2006)

Adamczyk, Z., Zembala, M., Siwek, B., Warszyński, P.: J. Colloid Interface Sci. 140, 123-124 (1990)

Adamczyk, Z., Senger, B., Voegel, J.C., Schaaf, P.: J. Chem. Phys. 110, 3118 (1999)

Adamczyk, Z., Warszyński, P., Zembala, M.: Bull. Pol. Acad. Chem. 47, 239-258 (1999)

Adamczyk, Z., Zembala, M., Michna, A.: J. Colloid Interface Sci. 303, 353-364 (2006)

Adamczyk, Z., Michna, A., Szaraniec, M., Bratek, A., Barbasz, J.: J. Colloid Interface Sci. 313, 86-96 (2007)

Adamczyk, Z., Sadlej, K., Wajnryb, E., Nattich, M., Ekiel-Jeżewska, M.L., Bławzdziewicz, J.: Adv. Colloid Interface Sci. 153, 1-29 (2010)

Adamczyk, Z., Barbasz, J., Cieśla, M.: Langmuir (2010, in press)

Adamczyk, Z., Zaucha, M., Zembala, M.: Langmuir 26, 9368 (2010)

Agnihotri, A., Siedlecki, C.A.: Langmuir 20, 8846 (2004)

Buijs, J., van den Berg, P.A.W., Lichtenbelt, J.W.Th., Norde, W., Lyklema, J.: J. Colloid Interface Sci. 178, 594-605 (1996)

Buijs, J., White, D.D., Norde, W.: Colloids Surf. B, Biointerfaces 8, 239-249 (1997)

Choi, K.H., Friedt, J.M., Frederix, F., Campitelli, A., Borghs, G.: Appl. Phys. Lett. 81, 1335-1337 (2002)

Elgersma, A.V., Zsom, R.L.J., Lyklema, J., Norde, W.: Colloid Surf. A 65, 17-28 (1992)

Etheve, J., Dejardin, P.: Langmuir 18, 1777-1785 (2002)

Hall, C.E., Slayter, H.S.J.: Biophys. Biochem. Cytol. 5, 11 (1959)

Hayes, R.A., Biehmer, M.R., Fokkink, L.G.J.: Langmuir 15, 2865 2870 (1999)

Hinrichsen, E.L., Feder, J., Jossang, T.: J. Stat. Phys. 44, 793 (1986)

Jin, X., Wang, N.H.L., Tarjus, G., Talbot, J.: J. Phys. Chem. 97, 42564258 (1993)

Marchin, K.L., Berrie, C.L.: Langmuir 19, 9883-9888 (2003)

Melmsten, M.: J. Colloid Interface Sci. 166, 333-342 (1994)

Norde, W., Rouwendal, E.: J. Colloid Interface Sci. 139, 169-176 (1990)

Ortega-Vinuesa, J.L., Tengvall, P., Lundstrom, I.: Thin Solid Films 325, 257 (1998)

Ramsden, J.J.: Q. Rev. Bipohys. 27, 41-105 (1993)

Reisch, A., Voegel, J.C., Gonthier, E., Decher, G., Senger, B., Schaaf, P., Mésini, P.J.: Langmuir 25, 3610-3617 (2009)

Scales, P.J., Grieser, F., Healy, T.W.: Langmuir 6, 582-589 (1990)

Scales, P.J., Grieser, F., Healy, T.W.: Langmuir 8, 965-974 (1992)

Schaaf, P., Talbot, J.: J. Chem. Phys. 91, 4401 (1989)

Sit, P.S., Marchant, R.E.: Thromb. Haemost. 82, 1053-1060 (1999)

Toscano, A., Santore, M.M.: Langmuir 22, 2588 (2006)

van Wagenen, R.A., Andrade, J.D.: J. Colloid Interface Sci. 76, 305314 (1980)

Vasina, E.N., Dejardin, P.: Langmuir 20, 8699-8706 (2004)

Veklich, Y.I., Gorkun, O.V., Medved, L.V., Nieuwenhuizen, W., Weisel, J.W.: J. Biol. Chem. 268, 13577-13585 (1993)

Wasilewska, M., Adamczyk, Z., Jachimska, B.: Langmuir 25, 369 (2009)

Wertz, Ch.F., Santore, M.M.: Langmuir 17, 3006-3010 (2001) 
Yoon, J.Y., Park, H.Y., Kim, J.H., Kim, W.S.: J. Colloid Interface Sci. 177, 613-620 (1996)

Zembala, M., Adamczyk, Z.: Langmuir 16, 1593-1601 (2000)

Zembala, M., Dejardin, P.: Colloids Surf. B 3, 119-129 (1994)
Zembala, M., Voegel, J.C., Schaaf, P.: Langmuir 14, 2167-2173 (1998)

Zembala, M., Adamczyk, Z., Warszyński, P.: Colloid Surf. A 222, 329_ 339 (2003) 